\title{
Matzoh ball soup revisited: the boundary regularity issue
}

\section{Rolando Magnaninia*† and Shigeru Sakaguchib}

\section{Communicated by Y. Xu}

We consider nonlinear diffusion equations of the form $\partial_{t} u=\Delta \phi(u)$ in $\mathbb{R}^{N}$ with $N \geq 2$. When $\phi(s) \equiv s$, this is just the heat equation. Let $\Omega$ be a domain in $\mathbb{R}^{N}$, where $\partial \Omega$ is bounded and $\partial \Omega=\partial\left(\mathbb{R}^{N} \backslash \bar{\Omega}\right)$. We consider the initial-boundary value problem, where the initial value equals zero and the boundary value equals 1 , and the Cauchy problem where the initial data is the characteristic function of the set $\Omega^{c}=\mathbb{R}^{N} \backslash \Omega$. We settle the boundary regularity issue for the characterization of the sphere as a stationary level surface of the solution $u$ :, no regularity assumption is needed for $\partial \Omega$. Copyright $\odot 2011$ John Wiley \& Sons, Ltd.

Keywords: nonlinear diffusion; heat equation; initial-boundary value problem; Cauchy problem; initial behavior; stationary level surface; sphere

\section{Introduction}

Let $\Omega$ be a domain in $\mathbb{R}^{N}(N \geq 2)$. Let $\phi: \mathbb{R} \rightarrow \mathbb{R}$ satisfy

$$
\phi \in C^{2}(\mathbb{R}), \quad \phi(0)=0, \text { and } 0<\delta_{1} \leq \phi^{\prime}(s) \leq \delta_{2} \text { for } s \in \mathbb{R},
$$

where $\delta_{1}, \delta_{2}$ are positive constants. Consider the unique bounded solution $u=u(x, t)$ of either the initial-boundary value problem

$$
\begin{aligned}
& \partial_{t} u=\Delta \phi(u) \text { in } \Omega \times(0,+\infty), \\
& u=1 \text { on } \partial \Omega \times(0,+\infty), \\
& u=0 \text { on } \Omega \times\{0\},
\end{aligned}
$$

or the Cauchy problem

$$
\partial_{t} u=\Delta \phi(u) \text { in } \mathbb{R}^{N} \times(0,+\infty) \text { and } u=\chi_{\Omega^{c}} \text { on } \mathbb{R}^{N} \times\{0\}
$$

here, $\chi_{\Omega^{c}}$ denotes the characteristic function of the set $\Omega^{c}=\mathbb{R}^{N} \backslash \Omega$. (As a solution, $u$ of problem (1.2)-(1.4), we mean a classical solution belonging to $C^{2,1}(\Omega \times(0,+\infty)) \cap L^{\infty}(\Omega \times(0,+\infty)) \cap C^{0}(\bar{\Omega} \times(0,+\infty))$ and such that $u(\cdot, t) \rightarrow 0$ in $L_{\text {loc }}^{1}(\Omega)$ as $t \rightarrow 0$; similarly, a solution of problem (1.5) is a classical solution belonging to $C^{2,1}\left(\mathbb{R}^{N} \times(0,+\infty)\right) \cap L^{\infty}\left(\mathbb{R}^{N} \times(0,+\infty)\right)$ and such that $u(\cdot, t) \rightarrow \chi \Omega^{c}(\cdot)$ in $L_{\text {loc }}^{1}\left(\mathbb{R}^{N}\right)$ as $t \rightarrow 0$. Note that the uniqueness of the solution of either problem (1.2)-(1.4) or problem (1.5) follows from the comparison principle, as shown in [1, Theorem A.1].)

In Theorem A in the succeeding texts, for the reader's convenience, we recall a nonlinear version of an asymptotic formula-proved by Varadhan in [2] for the linear case-that was proved in [3, Theorem 1.1 and Theorem 4.1] and [1, Theorem 2.1 and Remark 2.2]. To this aim, we define $\Phi:(0, \infty) \rightarrow \mathbb{R}$ by

$$
\Phi(s)=\int_{1}^{s} \frac{\phi^{\prime}(\xi)}{\xi} \mathrm{d} \xi \quad(s>0)
$$

${ }^{a}$ Dipartimento di Matematica U. Dini, Università di Firenze, viale Morgagni 67/A, 50134 Firenze, Italy

${ }^{b}$ Department of Applied Mathematics, Graduate School of Engineering, Hiroshima University, Higashi-Hiroshima, 739-8527, Japan

*Correspondence to: Rolando Magnanini, Dipartimento di Matematica U. Dini, Università di Firenze, viale Morgagni 67/A, 50134 Firenze, Italy.

† E-mail:magnanin@math.unifi.it 
(note that if $\phi(s) \equiv s$, then $\Phi(s)=\log s$ ) and let $\mathrm{d}=\mathrm{d}(x)$ be the distance function given by

$$
\mathrm{d}(x)=\operatorname{dist}(x, \partial \Omega) \text { for } x \in \Omega \text {. }
$$

Theorem A

$([1,3])$ Let $u$ be the solution of either problem (1.2)-(1.4) or problem (1.5). Under the assumption that $\partial \Omega=\partial\left(\mathbb{R}^{N} \backslash \bar{\Omega}\right)$,

$$
-4 t \Phi(u(x, t)) \rightarrow \mathrm{d}(x)^{2} \text { as } t \rightarrow 0^{+} \text {uniformly on every compact set in } \Omega \text {. }
$$

The assumption that $\partial \Omega=\partial\left(\mathbb{R}^{N} \backslash \bar{\Omega}\right)$ is general. For example, it holds for Lipschitz domains.

A conjecture, posed by Klamkin [4] and referred to by Zalcman [5] as the Matzoh ball soup was settled affirmatively by Alessandrini $[6,7]$. In [7], when $\phi(s) \equiv s$ and $\Omega$ is bounded, under the assumption that every point of $\partial \Omega$ is regular with respect to the Laplacian, it was proved that if all the spatial level surfaces of the solution $u$ of problem (1.2)-(1.4) are invariant with time then $\Omega$ must be a ball. The proof requires assuming that infinitely many level surfaces of $u$ are invariant with time. Here, we remark that the values of $u$ vary with time on its spatial level surfaces.

In $[3,8,9]$, we proved symmetry results for solutions of the problems (1.2)-(1.4) and (1.5), which admit a time-invariant level surface. Those results were obtained under classical regularity assumptions on the domains at stake. In the present paper, with the aid of Theorem A and Theorem B in the succeeding texts, we show that such results also hold under very general assumptions.

The following theorem removes the hypotheses made in [3, Theorem 1.2 and Theorem 1.3] that $\partial \Omega$ and $\partial D$ be $C^{2}$-smooth.

Theorem 1.1

Let $u$ be the unique bounded solution of either problem (1.2)-(1.4) or problem (1.5). Suppose that $\partial \Omega$ is bounded and $\partial \Omega=\partial\left(\mathbb{R}^{N} \backslash \bar{\Omega}\right)$.

Let $D$ be a $C^{1}$ domain, with bounded boundary $\partial D$, satisfying $\bar{D} \subset \Omega$. Then the following statements hold.

1. If there exists a function $a:(0,+\infty) \rightarrow(0,+\infty)$ satisfying

$$
u(x, t)=a(t) \text { for every }(x, t) \in \partial D \times(0,+\infty),
$$

then $\partial \Omega$ must be a sphere.

2. If $D$ is unbounded and for each connected component $\Gamma$ of $\partial D$ there exists a function $a_{\Gamma}:(0,+\infty) \rightarrow(0,+\infty)$ satisfying

$$
u(x, t)=a_{\Gamma}(t) \text { for every }(x, t) \in \Gamma \times(0,+\infty),
$$

then $\partial \Omega$ must be a sphere.

The next theorem concerns results obtained in $[3,8,9]$, and in particular, [3, Theorem 2.1]; we prove that they hold for a general domain $\Omega$, without assuming the exterior sphere condition on $\Omega$.

Theorem 1.2

Let $\phi(s) \equiv s$ and let $u$ be the unique bounded solution of either problem (1.2)-(1.4) or problem (1.5). Suppose that $\partial \Omega$ is bounded and $\partial \Omega=\partial\left(\mathbb{R}^{N} \backslash \bar{\Omega}\right)$.

Let $D$ be a domain, with bounded boundary $\partial D$, satisfying $\bar{D} \subset \Omega$, and let $\Gamma$ be a connected component of $\partial D$ satisfying

$$
\operatorname{dist}(\Gamma, \partial \Omega)=\operatorname{dist}(\partial D, \partial \Omega) \text {. }
$$

Suppose that $D$ satisfies the interior cone condition on $\Gamma$.

If there exists a function $a:(0,+\infty) \rightarrow(0,+\infty)$ satisfying

$$
u(x, t)=a(t) \text { for every }(x, t) \in \Gamma \times(0,+\infty),
$$

then $\partial \Omega$ must be either a sphere or the union of two concentric spheres.

We sketch the main features of the proof of Assertion (1) of Theorem 1.1. By Theorem A, there exists a number $R>0$ such that $\mathrm{d}(x)=R$ for all $x \in \partial D$, and hence, because $\partial D$ is of class $C^{1}$, we can conclude that $\Omega$ is the union of $D$ and all the open balls $B_{R}(x)$ of radius $R$ centered at points $x \in \partial D$. Thanks to this remark, we can apply the method of moving planes directly to either $D$ or $\mathbb{R}^{N} \backslash \bar{D}$ (in [3, Theorem 1.2 and Theorem 1.3], we applied it to either $\Omega$ or $\mathbb{R}^{N} \backslash \bar{\Omega}$, instead); for this reason, we do not need the smoothness of $\partial \Omega$. The proof of Assertion (2) of Theorem 1.1 runs similarly.

Eventually, Theorem 1.1 is proved by the method of moving planes, and hence, the following problem is open: When $D$ is bounded with disconnected boundary and for each connected component $\Gamma$ of $\partial D$, there exists a function $a_{\Gamma}:(0,+\infty) \rightarrow(0,+\infty)$ satisfying Equation (1.9), must $\partial \Omega$ be a sphere? Of course, it is assumed that $a_{\Gamma}$ 's are different for at least two components.

The removal of the exterior sphere condition on $\Omega$ in Theorem 1.2 relies on [1, Theorem 1.1 and Remark 1.2], that we summarize in Theorem B for later use.

Theorem B

([1, Theorem 1.1 and Remark 1.2]) Let $x_{0} \in \Omega$ and assume that the open ball $B_{R}\left(x_{0}\right)$ is contained in $\Omega$ and such that $\overline{B_{R}\left(x_{0}\right)} \cap \partial \Omega=\left\{y_{0}\right\}$ for some $y_{0} \in \partial \Omega$. Suppose that $\partial \Omega$ is of class $C^{2}$ in a neighborhood of the point $y_{0}$. 
Let $u$ be the solution of either problem (1.2)-(1.4) or problem (1.5). Then we have

$$
\lim _{t \rightarrow 0^{+}} t^{-\frac{N+1}{4}} \int_{B_{R}\left(x_{0}\right)} u(x, t) \mathrm{d} x=c(\phi, N)\left\{\prod_{j=1}^{N-1}\left(\frac{1}{R}-\kappa_{j}\left(y_{0}\right)\right)\right\}^{-\frac{1}{2}} .
$$

Here, $\kappa_{1}\left(y_{0}\right), \ldots, \kappa_{N-1}\left(y_{0}\right)$ denote the principal curvatures of $\partial \Omega$ at $y_{0}$ with respect to the inward normal direction to $\partial \Omega$ and $c(\phi, N)$ is a positive constant depending only on $\phi$ and $N$-of course, $c(\phi, N)$ depends on the problems (1.2)-(1.4) or (1.5).

When $\kappa_{j}\left(y_{0}\right)=\frac{1}{R}$ for some $j \in\{1, \cdots, N-1\}$, Equation (1.12) holds by setting the right-hand side to $+\infty$ (notice that $\kappa_{j}\left(y_{0}\right) \leq 1 / R$ always holds for all $j$ 's).

By this theorem and the balance law also used in $\left[3\right.$, Theorem 2.1] and $[8,9]$, first, we can begin with inferring that $\sum_{j=1}^{N-1}\left(\frac{1}{R}-\kappa_{j}\right)$ equals a positive constant on some portion of the boundary; and hence, analyticity of $\Gamma$ helps us extend such an equality to the whole connected component of $\partial \Omega$ parallel to $\Gamma$.

Sections 2 and 3 are devoted to the proofs of Theorems 1.1 and 1.2, respectively. In the Appendix, for the reader's convenience, we give a proof of Theorem $B$ for the heat equation under the assumption that $\max _{1 \leq j \leq N-1} \kappa_{j}\left(y_{0}\right)<\frac{1}{R}$. Then the proof of Theorem 1.2 will be self-contained.

\section{Proof of Theorem 1.1}

Let us prove Assertion (1) first. We start with a lemma.

Lemma 2.1

Under the assumptions of Assertion (1) of Theorem 1.1, there exists a number $R>0$ such that

$$
\mathrm{d}(x)=R \text { for all } x \in \partial D \text { and } \Omega=D \cup \bigcup_{x \in \partial D} B_{R}(x)=\left\{y \in \mathbb{R}^{N}: \operatorname{dist}(y, \bar{D})<R\right\} \text {. }
$$

Proof

Theorem $\mathrm{A}$ and Equation (1.8) imply that there exists a number $R>0$ such that $\mathrm{d}(x)=R$ for all $x \in \partial D$, which in turn immediately gives us the inclusion

$$
\Omega \supset D \cup \bigcup_{x \in \partial D} B_{R}(x)
$$

We observe that

$$
D \cup \bigcup_{x \in \partial D} B_{R}(x)=\left\{y \in \mathbb{R}^{N}: \operatorname{dist}(y, \bar{D})<R\right\}
$$

Let us show the converse inclusion of Formula (2.2). Because $\partial D$ is of class $C^{1}$, from the first part of Assertion (2.1) we have

$$
\overline{B_{R}(x)} \cap \partial \Omega=\{y(x)\} \text { and } B_{R}(y(x)) \cap D=\varnothing \text { for every } x \in \partial D,
$$

where $y(x)=x+R v_{\partial D}(x)$ and $v_{\partial D}(x)$ denotes the unit outward normal vector to $\partial D$ at $x \in \partial D$. Then it follows that

$$
\left\{y \in \mathbb{R}^{N}: \operatorname{dist}(y, \bar{D})=R\right\}=\{y(x): x \in \partial D\} \subset \partial \Omega .
$$

By Formulas (2.2) and (2.3),

$$
\left\{y \in \mathbb{R}^{N}: \operatorname{dist}(y, \bar{D})<R\right\} \subset \Omega \text {. }
$$

Observe that

$$
\partial\left\{y \in \mathbb{R}^{N}: \operatorname{dist}(y, \bar{D})<R\right\}=\left\{y \in \mathbb{R}^{N}: \operatorname{dist}(y, \bar{D})=R\right\} .
$$

Because $\Omega$ is a domain, in view of Formulas (2.5), (2.6) and (2.7), we conclude that

$$
\left\{y \in \mathbb{R}^{N}: \operatorname{dist}(y, \bar{D})=R\right\}=\partial \Omega \text { and }\left\{y \in \mathbb{R}^{N}: \operatorname{dist}(y, \bar{D})<R\right\}=\Omega,
$$

which yields the converse inclusion of Formula (2.2).

Let $\ell$ be a unit vector in $\mathbb{R}^{N}, \lambda \in \mathbb{R}$, and let $\pi_{\lambda}$ be the hyperplane $x \cdot \ell=\lambda$. 
Set $D_{\lambda}=\{x \in D: x \cdot \ell>\lambda\}$ and $\Omega_{\lambda}=\{x \in \Omega: x \cdot \ell>\lambda\}$, and denote by $D_{\lambda}^{\prime}$ and $\Omega_{\lambda}^{\prime}$ the reflection of $D_{\lambda}$ and $\Omega_{\lambda}$ in the plane $\pi_{\lambda}$, respectively.

Under the assumptions of Assertion (1) of Theorem 1.1, if $D_{\lambda}^{\prime} \subset D$, then $\Omega_{\lambda}^{\prime} \subset \Omega$.

Proof

Because $D_{\lambda}^{\prime} \subset D$, then also the set $D_{\text {sym }}=D_{\lambda} \cup\left(\pi_{\lambda} \cap D\right) \cup D_{\lambda}^{\prime}$ is contained in $D$. Thus, by Lemma 2.1, there holds that

$$
D_{\text {sym }} \cup \bigcup_{x \in \partial D_{\text {sym }}} B_{R}(x) \subset D \cup \bigcup_{x \in \partial D} B_{R}(x)=\Omega,
$$

and hence, $\Omega_{\lambda}^{\prime} \subset \Omega$.

We can now complete the proof of Assertion (1). Lemma 2.2 allows us to apply the method of moving planes, instead of to either $\Omega$ or $\mathbb{R}^{N} \backslash \bar{\Omega}$ as in the proof of [3, Theorem 1.2], directly to either $D$ or $\mathbb{R}^{N} \backslash \bar{D}$. Apart from this difference, the proof runs with the same arguments used in [3, Theorem 1.2]. It is worth noticing that, by [10, Section 5.2], the method of moving planes is applicable to $C^{1}$ domains, as $D$ is assumed to be.

The proof of Assertion (2) is similar to that of [3, Theorem 1.3].

\section{Proof of Theorem 1.2}

We recall that $D$ satisfies the interior cone condition with respect to $\Gamma$, if for every $x \in \Gamma$, there exists a finite right spherical open cone $K_{x}$ with vertex $x$ such that $K_{x} \subset D$ and $\overline{K_{x}} \cap \partial D=\{x\}$.

In view of the proof of [3, Theorem 2.1], Theorem 1.2 directly follows from the following lemma-note that this holds for general domains $\Omega$, including the case in which their boundaries are unbounded.

Lemma 3.1

Under the assumptions of Theorem 1.2, the following assertions hold, even if $\partial \Omega$ is unbounded.

1. There exists a number $R>0$ such that $\mathrm{d}(x)=R$ for every $x \in \Gamma$;

2. $\Gamma$ is a real analytic hypersurface;

3. there exists a connected component $\gamma$ of $\partial \Omega$, that is also a real analytic hypersurface, such that the mapping $\gamma \ni \xi \mapsto x(\xi) \equiv$ $\xi+R v(\xi) \in \Gamma$, where $v(\xi)$ is the inward unit normal vector to $\partial \Omega$ at $\xi \in \gamma$, is a diffeomorphism; in particular, $\gamma$ and $\Gamma$ are parallel hypersurfaces at distance $R$;

4. it holds that

$$
\max _{1 \leq j \leq N-1} \kappa_{j}(\xi)<\frac{1}{R} \text { for every } \xi \in \gamma
$$

where $\kappa_{1}(\xi), \cdots, \kappa_{N-1}(\xi)$ are the principal curvatures of $\partial \Omega$ at $\xi \in \gamma$ with respect to the inward unit normal vector to $\partial \Omega$;

5. there exists a number $c>0$ such that

$$
\prod_{j=1}^{N-1}\left(\frac{1}{R}-\kappa_{j}(\xi)\right)=c \quad \text { for every } \xi \in \gamma
$$

Remark

We emphasize a new important fact in this lemma: here, we do not assume the exterior sphere condition on $\Omega$, that was needed in previous papers $([3,8,9])$ to show that $\gamma$ and $\Gamma$ are parallel hypersurfaces. Thus, for example, with the aid of this lemma, we can remove the exterior-sphere-condition assumption from all the theorems [8, Theorem 1.1], [9, Theorem 3.1] and [3, Theorem 2.1], and obtain very general characterizations of the sphere in terms of stationary isothermic surfaces.

Therefore, the occurrence of a stationary isothermic surface is a very strong requirement indeed.

Proof

Proof of Lemma 3.1. First of all, Assertion (1) follows from Equation (1.11) and Theorem A. Assertion (2) follows from almost the same argument as in (ii) of Lemma 3.1 of [8]. Because here we also deal with the Cauchy problem and $\partial \Omega$ is not necessarily bounded, for the reader's convenience we give a proof by using Theorem A directly, instead of dealing with the Laplace transform of the solution as in [8]. Besides Theorem A, we use the balance law with respect to stationary critical points of the solution, the interior cone condition of $D$ together with Equations (1.10), (1.11), and Assertion (1).

It suffices to show that, for every point $x \in \Gamma$, there exists a time $t>0$ such that $\nabla u(x, t) \neq 0$; then, Assertion (2) follows from Equation (1.11), analyticity of $u$ with respect to the space variable, and the implicit function theorem. We use a balance law with respect to stationary critical points of the caloric functions stated as follows (see [8] for a proof): Let $G$ be a domain in $\mathbb{R}^{N}$. For $x_{0} \in G$, a solution $v=v(x, t)$ of the heat equation in $G \times(0,+\infty)$ is such that $\nabla v\left(x_{0}, t\right)=0$ for every $t>0$ if and only if

$$
\int_{\partial B_{r}\left(x_{0}\right)}\left(x-x_{0}\right) v(x, t) d S_{x}=0 \text { for every }(r, t) \in\left[0, \operatorname{dist}\left(x_{0}, \partial G\right)\right) \times(0,+\infty) .
$$


Assume by contradiction that there exists a point $x_{0} \in \Gamma$ such that $\nabla u\left(x_{0}, t\right)=0$ for every $t>0$. By Equation (3.3), we can infer that

$$
\int_{\partial B_{r}\left(x_{0}\right)}\left(x-x_{0}\right) u(x, t) d S_{X}=0 \text { for every }(r, t) \in(0, R) \times(0,+\infty) .
$$

Here, let us choose $r=\frac{R}{2}$.

On the other hand, because $D$ satisfies the interior cone condition, there exists a finite right spherical open cone $K$ with vertex at $x_{0}$ such that $K \subset D$ and $\bar{K} \cap \partial D=\left\{x_{0}\right\}$. By translating and rotating if needed, we can suppose that $x_{0}=0$ and that $K$ is the set $\left\{x \in B_{\rho}(0): x_{N}<-|x| \cos \theta\right\}$, where $\rho \in\left(0, \frac{R}{2}\right)$ and $\theta \in\left(0, \frac{\pi}{2}\right)$.

Because $K \subset D$ and $\bar{K} \cap \partial D=\{0\}$, Assertion (1) and Equation (1.10) imply that

$$
\mathrm{d}(x)>R \text { for every } x \in K \text {. }
$$

Let us set

$$
V_{s}=\left\{x \in \partial B_{s}(0): x_{N} \geq s \sin \theta\right\} \text { for } s>0 .
$$

Then

$$
\partial \Omega \cap \partial B_{R}(0) \subset V_{R}
$$

because, otherwise, there would be a point in $K$ contradicting Assertion (3.5).

Thus, from Assertion (3.7), it follows that we can choose a small number $\delta>0$ such that

$$
\mathrm{d}(x) \geq \frac{R}{2}+2 \delta \text { for every } x \in \partial B_{\frac{R}{2}}(0) \cap\left\{x_{N} \leq 0\right\} .
$$

Because, by Theorem $\mathrm{A},-4 t \log u(x, t)$ converges uniformly on $\partial B_{\frac{R}{2}}(0)$ to $\mathrm{d}(x)^{2}$ as $t \rightarrow 0^{+}$, we can choose $t^{*}>0$ such that

$$
\left|-4 t \log u(x, t)-\mathrm{d}(x)^{2}\right|<\delta^{2} \text { for every }(x, t) \in \partial B_{\frac{R}{2}}(0) \times\left(0, t^{*}\right) \text {. }
$$

This latter inequality, together with Assertions (3.6), (3.7), and (3.8), gives, for every $t \in\left(0, t^{*}\right)$, the following two estimates:

$$
\begin{gathered}
\int_{\partial B_{\frac{R}{2}}(0) \cap\left\{x_{N} \leq 0\right\}} x_{N} u(x, t) \mathrm{d} S_{X} \geq-\frac{R}{4} e^{-\frac{1}{4 t}\left(\frac{R^{2}}{4}+2 R \delta+3 \delta^{2}\right)} \mathcal{H}^{N-1}\left(\partial B_{\frac{R}{2}}(0)\right), \\
\int_{V_{\frac{R}{2}} \cap \overline{\Omega_{\frac{R}{2}+\delta}}} x_{N} u(x, t) \mathrm{d} S_{X} \geq \frac{R}{2} \sin \theta e^{-\frac{1}{4 t}\left(\frac{R^{2}}{4}+R \delta+2 \delta^{2}\right)} \mathcal{H}^{N-1}\left(V_{\frac{R}{2}} \cap \overline{\Omega_{\frac{R}{2}}+\delta}\right) .
\end{gathered}
$$

Here $\mathcal{H}^{N-1}(\cdot)$ denotes the $(N-1)$-dimensional Hausdorff measure and $\Omega_{\frac{R}{2}+\delta}$ is defined by

$$
\Omega_{\frac{R}{2}+\delta}=\left\{x \in \Omega: \mathrm{d}(x)<\frac{R}{2}+\delta\right\} .
$$

A consequence of Inequalities (3.9) and (3.10) is that, for every $t \in\left(0, t^{*}\right)$,

$$
\begin{aligned}
& \int_{\partial B_{\frac{R}{2}}(0)} x_{N} u(x, t) \mathrm{d} S_{X} \\
\geq & \int_{V_{\frac{R}{2}} \cap \bar{\Omega} \frac{R}{2}+\delta} x_{N} u(x, t) \mathrm{d} S_{X}+\int_{\partial B_{\frac{R}{2}}(0) \cap\left\{x_{N} \leq 0\right\}} x_{N} u(x, t) \mathrm{d} S_{X} \\
\geq & \frac{R}{4} e^{-\frac{1}{4 t}\left(\frac{R^{2}}{4}+R \delta+2 \delta^{2}\right)}\left[2 \sin \theta \mathcal{H}^{N-1}\left(V_{\frac{R}{2}} \cap \overline{\Omega_{\frac{R}{2}+\delta}}\right)-e^{-\frac{1}{4 t}\left(R \delta+\delta^{2}\right)} \mathcal{H}^{N-1}\left(\partial B_{\frac{R}{2}}(0)\right)\right] .
\end{aligned}
$$

Therefore, we obtain a contradiction by observing that the first term of this chain of inequalities equals zero, by Equation (3.4) with $r=\frac{R}{2}$, whereas the last term can be made positive by choosing $t>0$ sufficiently small. This completes the proof of Assertion (2).

Now, based on Assertions (1) and (2), let us prove Assertions (3), (4), and (5) at the same time without assuming the exterior sphere condition on $\Omega$, as pointed out in the previous remark.

In view of Assertion (2), let $\nu_{\Gamma}(x)$ and $\hat{\kappa}_{1}(x), \cdots, \hat{\kappa}_{N-1}(x)$ be the unit outward normal vector to $\partial D$ at $x \in \Gamma$ and the principal curvatures of $\Gamma$ at $x \in \Gamma$ with respect to $\nu_{\Gamma}(x)$, respectively. Notice that, in view of Equation (1.10), Assertions (2) and (1) imply that 
Moreover, $\xi=x+R v_{\Gamma}(x)$, and in view of Assertions (1) and (3.11), comparing the principal curvatures at $x$ of $\Gamma$ with those of the sphere $\partial B_{R}(\xi)$ yields that

$$
\max _{1 \leq j \leq N-1} \hat{\kappa}_{j}(x) \leq \frac{1}{R} \quad \text { for every } x \in \Gamma
$$

Because $\Gamma$ is a connected component of $\partial D, \Gamma$ is oriented and $\Gamma$ divides $\mathbb{R}^{N}$ into two domains. Let $E$ be the one of them that does not intersect $D$. By Assertion (1) and Equation (1.10), $E \cap\left(\mathbb{R}^{N} \backslash \bar{\Omega}\right)$ contains a point, say, $z$. Set $R_{0}=\operatorname{dist}(z, \Gamma)$. Then $R_{0}>R$ and there exists a point $p_{0} \in \Gamma$ such that $R_{0}=\left|z-p_{0}\right|$. Comparing the principal curvatures at $p_{0}$ of $\Gamma$ with those of the sphere $\partial B_{R_{0}}(z)$, yields that $\hat{\kappa}_{j}\left(p_{0}\right) \leq \frac{1}{R_{0}}<\frac{1}{R}$ for every $j=1, \ldots, N-1$. By continuity, there exists $\delta_{0}>0$ such that

$$
\max _{1 \leq j \leq N-1} \hat{\kappa}_{j}(x)<\frac{1}{R} \quad \text { for every } x \in \Gamma \cap \overline{B_{\delta_{0}}\left(p_{0}\right)}
$$

This fact guarantees that the mapping $B_{\delta_{0}}\left(p_{0}\right) \cap \Gamma \ni x \mapsto \xi(x) \equiv x+R v_{\Gamma}(x) \in \partial \Omega$ is a local diffeomorphism, that is, by letting $P_{0}=p_{0}+R v_{\Gamma}\left(p_{0}\right)$, we can find a neighborhood $U$ of $P_{0}$ in $\mathbb{R}^{N}$ such that the mapping $B_{\delta_{0}}\left(p_{0}\right) \cap \Gamma \ni x \mapsto \xi(x) \in U \cap \partial \Omega$ is a diffeomorphism. Moreover, because $\Gamma$ is a real analytic hypersurface because of Assertion (2), this diffeomorphism is also real analytic. Hence, $U \cap \partial \Omega$ is a portion of a real analytic hypersurface.

Notice that the principal curvatures $\kappa_{1}(\xi), \cdots, \kappa_{N-1}(\xi)$ of $\partial \Omega$ at $\xi \in U \cap \partial \Omega$ with respect to the inward unit normal vector to $\partial \Omega$ satisfy

$$
-\kappa_{j}(\xi(x))=\frac{\hat{\kappa}_{j}(x)}{1-R \hat{\kappa}_{j}(x)} \text { for every } j=1, \ldots, N-1 \text { and every } x \in \Gamma \cap B_{\delta_{0}}\left(p_{0}\right) .
$$

Therefore, because $1-R \kappa_{j}(\xi(x))=1 /\left(1-R \hat{\kappa}_{j}(x)\right)$, we see that Inequality (3.13) is equivalent to

$$
\max _{1 \leq j \leq N-1} \kappa_{j}(\xi)<\frac{1}{R} \quad \text { for every } \xi \in \bar{U} \cap \partial \Omega
$$

We now use another balance law with respect to stationary zeros of the caloric functions stated as follows (see [8] for a proof): Let $G$ be a domain in $\mathbb{R}^{N}$. For $x_{0} \in G$, a solution $v=v(x, t)$ of the heat equation in $G \times(0,+\infty)$ is such that $v\left(x_{0}, t\right)=0$ for every $t>0$ if and only if

$$
\int_{\partial B_{r}\left(x_{0}\right)} v(x, t) d S_{X}=0 \text { for every }(r, t) \in\left[0, \operatorname{dist}\left(x_{0}, \partial G\right)\right) \times(0,+\infty) .
$$

Let $P, Q \in U \cap \partial \Omega$ be two distinct points, and let $p, q \in B_{\delta_{0}}\left(p_{0}\right) \cap \Gamma$ be the points such that $\xi(p)=P$ and $\xi(q)=Q$. Then, by Assertion (3.11), we have

$$
\overline{B_{R}(p)} \cap \partial \Omega=\{P\} \text { and } \overline{B_{R}(q)} \cap \partial \Omega=\{Q\}
$$

Consider the function $v=v(x, t)$ defined by

$$
v(x, t)=u(x+p, t)-u(x+q, t) \text { for }(x, t) \in B_{R}(0) \times(0,+\infty)
$$

Because $v$ satisfies the heat equation and, by Equation (1.11), $v(0, t)=0$ for every $t>0$, it follows from Equation (3.15) that

$$
\int_{B_{R}(p)} u(x, t) \mathrm{d} x=\int_{B_{R}(q)} u(x, t) \mathrm{d} x \text { for every } t>0
$$

Therefore, by Theorem B and Inequality (3.14), multiplying both sides by $t^{-\frac{N+1}{4}}$ and letting $t \rightarrow 0^{+}$yield that

$$
\prod_{j=1}^{N-1}\left(\frac{1}{R}-\kappa_{j}(P)\right)=\prod_{j=1}^{N-1}\left(\frac{1}{R}-\kappa_{j}(Q)\right)
$$

Hence, it follows that there exists a constant $c>0$ such that

$$
\prod_{j=1}^{N-1}\left(\frac{1}{R}-\kappa_{j}(\xi)\right)=c \text { for every } \xi \in U \cap \partial \Omega
$$


Because $1-R \kappa_{j}(\xi(x))=1 /\left(1-R \hat{\kappa}_{j}(x)\right)$, we see that

$$
\prod_{j=1}^{N-1}\left(\frac{1}{R}-\hat{\kappa}_{j}(x)\right)=\frac{1}{c R^{2(N-1)}}(>0) \text { for every } x \in B_{\delta_{0}}\left(p_{0}\right) \cap \Gamma .
$$

Moreover, analyticity of $\Gamma$ yields that this equality holds also for every $x \in \Gamma$, and hence, by Inequality (3.12)

$$
\max _{1 \leq j \leq N-1} \hat{\kappa}_{j}(x)<\frac{1}{R} \quad \text { for every } x \in \Gamma \text {. }
$$

Therefore, with the aid of this strict inequality, by setting

$$
\gamma=\left\{\xi(x) \in \mathbb{R}^{N}: x \in \Gamma\right\}
$$

we see that the mapping $\Gamma \ni x \mapsto \xi(x) \equiv x+R v_{\Gamma}(x) \in \gamma$ is a real analytic diffeomorphism because of analyticity of $\Gamma$, and $\gamma$ is a connected component of $\partial \Omega$, which is a real analytic hypersurface. Because the mapping $\gamma \ni \xi \mapsto x(\xi) \equiv \xi+R v(\xi) \in \Gamma$ is the inverse mapping of the previous diffeomorphism, Assertion (3) holds. Assertion (4) follows from Inequality (3.17).

Finally, combining analyticity of $\gamma$ with Equation (3.16) yields Equation (3.2). The proof is complete.

\section{Appendix}

Here, for the reader's convenience, we give a proof of Theorem B for the heat equation provided $\underset{1 \leq j \leq N-1}{\max } \kappa_{j}\left(y_{0}\right)<\frac{1}{R}$ by using some idea and a geometric lemma from [11].

Proof of Theorem B for the heat equation provided $\max _{1 \leq j \leq N-1} \kappa_{j}\left(y_{0}\right)<\frac{1}{R}$.

Set $\phi(s) \equiv s$. We distinguish two cases:

$$
\text { (I) } \partial \Omega \text { is bounded and of class } C^{2} ; \quad \text { (II) } \partial \Omega \text { is otherwise. }
$$

Let us first show how we obtain case (II) once we have proved case (I). Indeed, we can find two $C^{2}$ domains, say $\Omega_{1}$ and $\Omega_{2}$, with bounded boundaries, and a ball $B_{\delta}\left(y_{0}\right)$ with the following properties: $\Omega_{1}$ and $\mathbb{R}^{N} \backslash \overline{\Omega_{2}}$ are bounded; $B_{R}\left(x_{0}\right) \subset \Omega_{1} \subset \Omega \subset \Omega_{2}$;

$$
B_{\delta}\left(y_{0}\right) \cap \partial \Omega \subset \partial \Omega_{1} \cap \partial \Omega_{2} \text { and } \overline{B_{R}\left(x_{0}\right)} \cap\left(\mathbb{R}^{N} \backslash \Omega_{i}\right)=\left\{y_{0}\right\} \text { for } i=1,2
$$

Let $u_{i}=u_{i}(x, t)(i=1,2)$ be the two bounded solutions of either problem (1.2)-(1.4) or problem (1.5), where $\Omega$ is replaced by $\Omega_{1}$ or $\Omega_{2}$, respectively. Because $\Omega_{1} \subset \Omega \subset \Omega_{2}$, it follows from the comparison principle that

$$
u_{2} \leq u \text { in } \Omega \times(0,+\infty) \text { and } u \leq u_{1} \text { in } \Omega_{1} \times(0,+\infty)
$$

Therefore, it follows that for every $t>0$

$$
t^{-\frac{N+1}{4}} \int_{B_{R}\left(x_{0}\right)} u_{2}(x, t) \mathrm{d} x \leq t^{-\frac{N+1}{4}} \int_{B_{R}\left(x_{0}\right)} u(x, t) \mathrm{d} x \leq t^{-\frac{N+1}{4}} \int_{B_{R}\left(x_{0}\right)} u_{1}(x, t) \mathrm{d} x .
$$

These two inequalities show that case (I) implies case (II).

Hereafter, we assume that $\partial \Omega$ is bounded and of class $C^{2}$. Let us consider the signed distance function $\mathrm{d}^{*}=\mathrm{d}^{*}(x)$ of $x \in \mathbb{R}^{N}$ to the boundary $\partial \Omega$ defined by

$$
d^{*}(x)=\left\{\begin{aligned}
\operatorname{dist}(x, \partial \Omega) & \text { if } x \in \Omega \\
-\operatorname{dist}(x, \partial \Omega) & \text { if } x \notin \Omega
\end{aligned}\right.
$$

Because $\partial \Omega$ is bounded and of class $C^{2}$, there exists a number $\rho_{0}>0$ such that $d^{*}(x)$ is $C^{2}$-smooth on a compact neighborhood $\mathcal{N}$ of the boundary $\partial \Omega$ given by

$$
\mathcal{N}=\left\{x \in \mathbb{R}^{N}:-\rho_{0} \leq \mathrm{d}^{*}(x) \leq \rho_{0}\right\}
$$

We write for $s>0$

$$
\Omega_{s}=\left\{x \in \Omega: d^{*}(x)<s\right\}\left(=\left\{x \in \mathbb{R}^{N}: 0<d^{*}(x)<s\right\}\right) .
$$


Introduce a function $F=F(\xi)$ defined for $\xi \in \mathbb{R}$ by

$$
F(\xi)=\frac{1}{2 \sqrt{\pi}} \int_{\xi}^{\infty} e^{-s^{2} / 4} d s
$$

Then $F$ satisfies

$$
\begin{aligned}
& F^{\prime \prime}+\frac{1}{2} \xi F^{\prime}=0 \text { and } F^{\prime}<0 \text { in } \mathbb{R} \\
& F(-\infty)=1, F(0)=\frac{1}{2}, \text { and } F(+\infty)=0
\end{aligned}
$$

For each $\varepsilon \in(0,1 / 4)$, we define two functions $F_{ \pm}=F_{ \pm}(\xi)$ for $\xi \in \mathbb{R}$ by

$$
F_{ \pm}(\xi)=F(\xi \mp 2 \varepsilon) .
$$

Then $F_{ \pm}$satisfies

$$
\begin{aligned}
& F_{ \pm}^{\prime \prime}+\frac{1}{2} \xi F_{ \pm}^{\prime}= \pm \varepsilon F_{ \pm^{\prime}}^{\prime} F_{ \pm}^{\prime}<0 \text { and } F_{-}<F<F_{+} \text {in } \mathbb{R}, \\
& F_{ \pm}(-\infty)=1, F_{ \pm}(0) \gtrless \frac{1}{2}, \text { and } F_{ \pm}(+\infty)=0 .
\end{aligned}
$$

By setting

$$
v_{ \pm}(x, t)=F_{ \pm}\left(t^{-\frac{1}{2}} d^{*}(x)\right) \text { for }(x, t) \in \mathbb{R}^{N} \times(0,+\infty)
$$

we can state

Lemma B. 1

For each $\varepsilon \in(0,1 / 4)$, there exists $t_{1, \varepsilon}>0$ satisfying

$$
( \pm 1)\left\{\left(v_{ \pm}\right)_{t}-\Delta v_{ \pm}\right\}>0 \quad \text { in } \mathcal{N} \times\left(0, t_{1, \varepsilon}\right] .
$$

Proof

A straightforward computation gives

$$
\left(v_{ \pm}\right)_{t}-\Delta v_{ \pm}=-\frac{1}{t}\left( \pm \varepsilon+\sqrt{t} \Delta \mathrm{d}^{*}\right) F_{ \pm}^{\prime} \quad \text { in } \mathcal{N} \times(0,+\infty)
$$

Then, for each $\varepsilon \in(0,1 / 4)$, by setting $t_{1, \varepsilon}=\left(\frac{\varepsilon}{2 M}\right)^{2}$, where $M=\max _{x \in \mathcal{N}}\left|\Delta d^{*}(x)\right|$, we complete the proof.

Set $\rho_{1}=\max \left\{2 R, \rho_{0}\right\}$. Let $u$ be the solution of either problem (1.2)-(1.4) or problem (1.5). By Theorem A, we have that

$$
-4 t \log u(x, t) \rightarrow \mathrm{d}^{*}(x)^{2} \text { as } t \rightarrow 0^{+} \text {uniformly on } \overline{\Omega_{\rho_{1}} \backslash \mathcal{N}} \text {. }
$$

Then, in view of this and the definition (B.3) of $v_{ \pm}$, we have

\section{Lemma B.2}

Let $u$ be the solution of either problem (1.2)-(1.4) or problem (1.5). There exist three positive constants $t_{0}, E_{1}$ and $E_{2}$ satisfying

$$
\max \left\{\left|v_{+}\right|,\left|v_{-}\right|,|u|\right\} \leq E_{1} e^{-\frac{E_{2}}{t}} \text { in } \overline{\Omega_{\rho_{1}} \backslash \mathcal{N}} \times\left(0, t_{0}\right]
$$

Proof

If we choose $t_{0} \in\left(0,\left(\frac{\rho_{0}}{4}\right)^{2}\right]$, then by Equation (B.3), we can show the desired inequalities for $v_{ \pm}$. As for $u$, by Theorem A, we can take $t_{0}>0$ such that

$$
\left|4 t \log u(x, t)+\mathrm{d}^{*}(x)^{2}\right|<\frac{1}{2} \rho_{0}^{2} \text { for }(x, t) \in \overline{\Omega_{\rho_{1}} \backslash \mathcal{N}} \times\left(0, t_{0}\right],
$$

and hence,

$$
u(x, t)<e^{-\frac{d^{*}(x)^{2}-\frac{1}{2} \rho_{0}^{2}}{4 t}} \text { for }(x, t) \in \bar{\Omega} \times\left(0, t_{0}\right]
$$

Because $\mathrm{d}^{*}(x) \geq \rho_{0}$ for $x \in \overline{\Omega_{\rho_{1}} \backslash \mathcal{N}}$, we get the desired inequality for $u$. 
By setting, for $(x, t) \in \mathbb{R}^{N} \times(0,+\infty)$,

$$
w_{ \pm}(x, t)=\left\{\begin{aligned}
2 v_{ \pm}(x, t) \pm 2 E_{1} e^{-\frac{E_{2}}{t}} & \text { for problem (1.2)-(1.4) } \\
(1 \pm \varepsilon) v_{ \pm}(x, t) \pm 2 E_{1} e^{-\frac{E_{2}}{t}} & \text { for problem (1.5) }
\end{aligned}\right.
$$

we have

Lemma B.3

Let $u$ be the solution of either problem (1.2)-(1.4) or problem (1.5). For each $\varepsilon \in(0,1 / 4)$, there exists $t_{\varepsilon}>0$ satisfying

$$
w_{-} \leq u \leq w_{+} \quad \text { in } \overline{\Omega_{\rho_{1}}} \times\left(0, t_{\varepsilon}\right]
$$

where $w_{ \pm}$are defined by Equation (B.5).

Proof

For each $\varepsilon \in(0,1 / 4)$, we set

$$
t_{2, \varepsilon}=\min \left\{t_{1, \varepsilon}, t_{0}\right\}
$$

Because $v_{+}, v_{-}$, and $u$ are all nonnegative, Lemma B.2 implies that

$$
w_{-} \leq u \leq w_{+} \quad \text { in } \overline{\Omega_{\rho_{1}} \backslash \mathcal{N}} \times\left(0, t_{2, \varepsilon}\right] .
$$

Let $u$ be the solution of problem (1.2)-(1.4). Observe that

$$
\begin{array}{ll}
w_{-} \leq u \leq w_{+} & \text {on } 2 \Omega \times\left(0, t_{2, \varepsilon}\right] \\
w_{-}=u=w_{+}=0 & \text { on }(\Omega \cap \mathcal{N}) \times\{0\} .
\end{array}
$$

Therefore, with the aid of the comparison principle and in view of Lemma B.1, Inequalities (B.7), (B.8), and Equation (B.9), we obtain Inequality (B.6) by setting $t_{\varepsilon}=t_{2, \varepsilon}$.

It remains to consider the solution $u$ of problem (1.5). In view of the fact that $F_{ \pm}(-\infty)=1$, there exists $t_{3, \varepsilon} \in\left(0, t_{2, \varepsilon}\right]$ such that

$$
w_{-}<u<w_{+} \text {on }(\partial \mathcal{N} \backslash \Omega) \times\left(0, t_{3, \varepsilon}\right] .
$$

Observe also that

$$
w_{-} \leq u \leq w_{+} \quad \text { on } \mathcal{N} \times\{0\}
$$

Therefore, with the aid of the comparison principle and in view of Lemma B.1, Inequalities (B.7), (B.10), and (B.11), we obtain Inequality (B.6) by setting $t_{\varepsilon}=t_{3, \varepsilon}$.

By writing

$$
\Gamma_{s}=\left\{x \in \Omega: d^{*}(x)=s\right\} \text { for } s>0,
$$

let us quote a geometric lemma from [11] adjusted to our situation.

Lemma B.4

([11, Lemma 2.1, p. 376]) If $\max _{1 \leq j \leq N-1} \kappa_{j}\left(y_{0}\right)<\frac{1}{R}$, then we have

$$
\lim _{s \rightarrow 0^{+}} s^{-\frac{N-1}{2}} \mathcal{H}^{N-1}\left(\Gamma_{s} \cap B_{R}\left(x_{0}\right)\right)=2^{\frac{N-1}{2}} \omega_{N-1}\left\{\prod_{j=1}^{N-1}\left(\frac{1}{R}-\kappa_{j}\left(y_{0}\right)\right)\right\}^{-\frac{1}{2}},
$$

where $\mathcal{H}^{N-1}$ is the standard $(N-1)$-dimensional Hausdorff measure, and $\omega_{N-1}$ is the volume of the unit ball in $\mathbb{R}^{N-1}$.

Because $\max _{1 \leq j \leq N-1} \kappa_{j}\left(y_{0}\right)<\frac{1}{R}$, we can use this lemma.

Lemma B.3 implies that for every $t \in\left(0, t_{\varepsilon}\right]$

$$
t^{-\frac{N+1}{4}} \int_{B_{R}\left(x_{0}\right)} w_{-} \mathrm{d} x \leq t^{-\frac{N+1}{4}} \int_{B_{R}\left(x_{0}\right)} u \mathrm{~d} x \leq t^{-\frac{N+1}{4}} \int_{B_{R}\left(x_{0}\right)} w_{+} \mathrm{d} x
$$

Also, with the aid of the co-area formula, we have

$$
\int_{B_{R}\left(x_{0}\right)} v_{ \pm} \mathrm{d} x=t^{\frac{N+1}{4}} \int_{0}^{2 R t^{-\frac{1}{2}}} F_{ \pm}(\xi) \xi^{\frac{N-1}{2}}\left(t^{\frac{1}{2}} \xi\right)^{-\frac{N-1}{2}} \mathcal{H}^{N-1}\left(\Gamma_{t^{\frac{1}{2}} \xi} \cap B_{R}\left(x_{0}\right)\right) \mathrm{d} \xi
$$

where $v_{ \pm}$is defined by Equation (B.3). 
First, we take care of problem (1.2)-(1.4). By Lebesgue's dominated convergence theorem, Lemma B.4 and Equation (B.13), we get

$$
\lim _{t \rightarrow 0^{+}} t^{-\frac{N+1}{4}} \int_{B_{R}\left(x_{0}\right)} w_{ \pm} \mathrm{d} x=2^{\frac{N-1}{2}} \omega_{N-1}\left\{\prod_{j=1}^{N-1}\left(\frac{1}{R}-\kappa_{j}\left(y_{0}\right)\right)\right\}^{-\frac{1}{2}} \int_{0}^{\infty} 2 F_{ \pm}(\xi) \xi^{\frac{N-1}{2}} \mathrm{~d} \xi .
$$

Moreover, again by Lebesgue's dominated convergence theorem, we see that

$$
\lim _{\varepsilon \rightarrow 0} \int_{0}^{\infty} 2 F_{ \pm}(\xi) \xi^{\frac{N-1}{2}} \mathrm{~d} \xi=\int_{0}^{\infty} 2 F(\xi) \xi^{\frac{N-1}{2}} \mathrm{~d} \xi
$$

Therefore, because $\varepsilon>0$ is arbitrarily small in the inequalities (B.12), it follows that Equation (1.12) holds true with

$$
c(\phi, N)=2^{\frac{N-1}{2}} \omega_{N-1} \int_{0}^{\infty} 2 F(\xi) \xi^{\frac{N-1}{2}} \mathrm{~d} \xi
$$

In the case of problem (1.5), the proof runs similarly by replacing $2 F_{ \pm}, 2 F$ with $(1 \pm \varepsilon) F_{ \pm}$, $F$, respectively, in Equations (B.14)-(B.16).

\section{Acknowledgements}

The main part of the present paper was written during a stay of the second author at the Department of Mathematics of the University of Florence. He gratefully acknowledges its hospitality. The authors would also like to thank the referees for their many valuable suggestions to improve clarity in several points.

This research was partially supported by a Grant-in-Aid for Scientific Research (B) ( $\$$ 20340031) of Japan Society for the Promotion of Science and by a Grant of the Italian MURST.

To Bob with gratitude.

\section{References}

1. Magnanini R, Sakaguchi S. Interaction between nonlinear diffusion and geometry of domain. arXiv:1009.6131v2, to appear in Journal of Differential Equations.

2. Varadhan SRS. On the behavior of the fundamental solution of the heat equation with variable coefficients. Communications on Pure and Applied Mathematics 1967; 20:431-455.

3. Magnanini R, Sakaguchi S. Nonlinear diffusion with a bounded stationary level surface. Annales de I'Institut Henri Poincaré - (C) Analyse Non Linéaire 2010; 27:937-952.

4. Klamkin MS. A physical characterization of a sphere (Problem 64-5*). SIAM Review 1964; 6:61.

5. Zalcman L. Some inverse problems of potential theory. Contemporary Mathematics 1987; 63:337-350.

6. Alessandrini G. Matzoh ball soup: a symmetry result for the heat equation. Journal d'Analyse Mathematique 1990; 54:229-236.

7. Alessandrini G. Characterizing spheres by functional relations on solutions of elliptic and parabolic equations. Applicable Analysis 1991; 40:251-261.

8. Magnanini R, Sakaguchi S. Matzoh ball soup: heat conductors with a stationary isothermic surface. Annals of Mathematics 2002; 156:931-946.

9. Magnanini R, Sakaguchi S. Stationary isothermic surfaces for unbounded domains. Indiana University Mathematics Journal 2007; 56:2723-2738.

10. Fraenkel LE. An Introduction to Maximum Principles and Symmetry in Elliptic Problems. Cambridge University Press: Cambridge, 2000.

11. Magnanini R, Sakaguchi S. Interaction between degenerate diffusion and shape of domain. Proceedings of the Royal Society of Edinburgh: Section A 2007; 137:373-388. 